\title{
The Alfven Wave Zoo
}

\author{
A. S. de Assis ${ }^{1}$, C. E. da Silva ${ }^{2}$, V. J. O. Werneck de Carvalho ${ }^{3}$ \\ ${ }^{1}$ Fluminense Federal University, Niteroi, RJ, Brasil \\ ${ }^{2}$ State University of Rio de Janeiro, Rio de Janeiro, RJ, Brasil \\ ${ }^{3}$ Departamento de Geopfísica, Universidade Federal Fluminense, Niterói, RJ, Brasil \\ Email: altairsouzadeassis@gmail.com, claudio.elias13@gmail.com,vinicius_werneck@id.uff.br
}

How to cite this paper: de Assis, A.S., da Silva, C.E. and Werneck de Carvalho, V.J.O. (2020) The Alfven Wave Zoo. Open Access Library Journal, 7: e6378.

https://doi.org/10.4236/oalib.1106378

Received: April 30, 2020

Accepted: June 27, 2020

Published: June 30, 2020

Copyright $\odot 2020$ by author(s) and Open Access Library Inc.

This work is licensed under the Creative Commons Attribution International License (CC BY 4.0).

http://creativecommons.org/licenses/by/4.0/ (c) (i) Open Access

\begin{abstract}
It has been shown that a variety of names are assigned to the original MHD Alfven wave derived originally by Hannes Alfven in the 40s (Nature 150, 405-406 (1942)), and those names are used due to the different magnetic geometries where the target plasma could be confined, that is, in laboratory, in fusion, in space, and in astrophysics, where one could use as working geometry systems such as cartesian, cylindrical, toroidal, dipolar, and even more complex ones. We also show that different names with no new dramatic new physics induce misleading information on what is new and relevant and what is old related to the considered wave mode. We also show that changing the confining geometry and the background plasma kinetic properties, the Alfven wave dispersion properties change accordingly and this change "induces" all types of non-necessary swarm of names for the same MHD shear Alfven wave derived previously by Alfven.
\end{abstract}

\section{Subject Areas}

Plasma Physics

\section{Keywords}

Alfven, Plasma Physics, Solar Physics, Solar Wind, Space Plasma Physics, Astrophysical Plasmas, Nuclear Fusion, Alfven Waves

\section{Introduction}

Long ago, Alfven first postulated (derived) the existence of Magneto-Hydrodynamic Waves-MHD waves-today called Alfven wave. For the history sake one can have a look at: reference [1].

Alfven (global or surface) wave is now very well accepted as a possible plasma

Eigen mode for magnetized plasmas, it can be excited/present in fusion plasmas 
or space/astrophysical plasmas. As it is very well known the Alfven wave is a MHD Eigen mode where the ions provide the necessary inertia and the magnetic field lines support the effective tension that creates a restoring force for any equilibrium perturbation. Those modes can be excited by twisting the magnetic field lines (by an imposed dc electric current, for instance) or by compressing the plasma. The lines and the plasma are frozen together and the neutralizing electrons plasma is considered one fluid with ions [1] [2] [3] [4].

In past Alfven waves were not very well accepted as a possible plasma mode.

At that time, the scarce plasma physics community did not accept this finding promptly. However, after the observation of those plasma modes in solar wind, laboratory and magnetosphere/ionosphere, and behaving exactly as Alfven had postulated, the Alfven wave became then a respectable reality. After that many names were assigned to this mode, in different plasma physics communities; in space, in astrophysics, in technology and in fusion, inducing sometimes the bad idea that a new kind of "Eigen" modes were discovered. But, they are no other mode, they are with no caveat the well-known Alfven's Alfven wave [1] [2] [3] [4] $[5]$.

Alfven derived his new wave mode, using the very basic MHD equations. With a more elaborate calculation than Alfven's original model, using for instance the plasma dielectric properties, any plasma mode can be obtained including the original Alfven wave.

In general, due to its intrinsic complicated dielectric properties plasmas can support infinite different Eigen modes. The scenarios get even more complicated in complex geometries such as toroidal and dipolar-as found in fusion machines, planetary magnetospheres and pulsar magnetospheres. Due to the intrinsic complications, the Alfven wave can be very diverse related to the properties of dispersivity, polarization and dissipation in different background plasmas, but it keeps being Alfven wave, with no caveat. Indeed, no new dramatic physics is found, just new geometric and thermal effects due to thermal dynamics and the curved background magnetic field; this curved magnetic field lines force the electrons and ions to follow curved trajectories and also permit particles to be trapped in magnetic traps.

In this paper we discuss the reason for so many names for the same MHD shear Alfven wave earlier derived by Alfven [6]-[31].

\section{Alfven Wave Zoo}

The importance to understand the Alfvenic plasma motions is of paramount importance to determine plasma stability, plasma heating, non-inductive current drive, and particle acceleration.

Therefore, before moving to any application Alfven wave-particle interaction processes, it is in order to discuss a bit more on what type of Alfven wave people are talking about and how they name it. This means that it is necessary to explore a little further the "animals" present in the Alfven wave's zoo. 
Moving now inside the Alfven wave's zoo, we learn that they can be by nature of two types: shear and compressional, but in structure they can be global or surface. All of them can be derived from MHD equations with appropriate boundary conditions - it means bounded plasma or infinite plasma-as Alfven did. In magnetospheres, for instance, all types of these modes are observed, of course with different names used such as Alfven wave, MHD Alfven wave, kinetic Alfven wave, shear Alfven wave, inertial Alfven wave, compressional Alfven wave, Alfven surface wave and electromagnetic ion cyclotron wave (EMICW). Those names are used depending on the characteristic of the local plasma: MHD (cold) or kinetic (lukewarm/warm/hot), current carrying or not current carrying, homogeneous or nonhomogeneous. Geometrically speaking, for practical purposes, one can access the Alfven Eigen modes via Cartesian, cylindrical, toroidal or dipolar systems, since the geometrical model matters, it means that this can introduce modifications at their dispersion relation and one should not confuse those novel properties induced by real new physical effects. Complex geometry can aloud, for instance, different Alfven Eigen modes to couple M. Mahajan [11].

The global Alfven wave also called current carrying Alfven wave, TAE and GAE, should also be observed in some regions of the current carrying Space/Solar/Astrophysical plasmas.

If one is interested to study the particle acceleration processes in plasmas, they need to access the dissipation (energy and momentum) aspects of Alfven waves, therefore it is necessary to introduce kinetic corrections into the Alfven wave dispersion relation, in order to show how the non-dissipative MHD modes can be dissipated via the collisionless Cherenkov damping (Landau damping and TTMP-Transit Time Magnetic Pumping). Those dissipations arise due to the Alfven wave kinetic coupling to plasma electrostic modes giving raise to wave electric field aligned with the ambient magnetic field.

The Landau damping is related to the wave electric field and the Transit-Time Magnetic Pumping due to the wave magnetic field. At the linear regime, the plasma electromagnetic field is small, and therefore can be considered a first order expansion parameter to expand at all equations of the MHD system. If the electromagnetic field has finite amplitude the quadratic terms plays important role for plasma heating, current drive, and particle acceleration. Plasma heating occurs due to the wave energy thermalization into the bulk plasma and particle acceleration are more related to only the resonant electrons. If the thermalization time is smaller than the acceleration time, perceptible acceleration will be observed [15]-[29].

In order to move further, it is interesting to understand a bit more why people use using so many different names for the same Alfven mode? Is this just due to the tradition in plasma physics to create names and more names meaning just exactly the same thing, physically speaking? The point is, there are indeed only two Alfven modes, that is, the shear mode and the compressional mode in different geometries and derived with/without kinetic corrections. They have 
nothing really physically new from the original Alfven's Alfven wave derived so many years ago. They are not a new Alfven wave so to say.

We can find in the literature all sorts of names for the Alfven shear mode: For instance, oblique Alfven wave, dispersive Alfven wave, Inertial Alfven wave, discrete Alfven wave, etc.. All of them refer to the same shear Alfven mode, of course in a certain plasma and frequency region particular conditions.

In order to move further with this conjecture, we consider a coordinate system in which $z$ is the direction of the ambient magnetic field. In our model the $x$, $y$, and $z$ coordinates correspond to the east west, north south, and vertical directions, respectively.

The general dispersion relation for the shear Alfven branch in homogenous uniformly magnetized plasmas is given by the elegant expression [12]:

$$
N^{2}=\frac{\in_{x x}}{\frac{k_{z}^{2}}{k^{2}}+\frac{k_{\perp}^{2}}{k^{2}} \frac{\epsilon_{x x}}{\in_{z z}}}
$$

where $N^{2}=\frac{k^{2} c^{2}}{\omega^{2}}, \in_{x x}, \in_{z z}, k$ and $c$ are the total refraction index, the $x x$ and $z z$ components of the dielectric tensor, the wave number, the speed of light and the wave frequency, respectively. This simple relation (1) allows two uncoupled independent modes, a shear mode and a compressible one.

This dispersion relation (Equation (1)) is as complicated or as complete as the dielectric tensor components, which are dependent on the plasma geometry and dimension models used. The model can be one fluid-MHD, two-fluids, $\mathrm{n}$-fluids, hybrid (fluid ions + kinetic electrons or vice versa), kinetic, cold, lukewarm, hot, low beta, high beta, homogeneous, non-homogeneous, relativistic, non-relativistic, cartesian, cypindrical, toroidal or dipolar-1D, 2D or 3D.

From all those models, we can derive all the different modes that are part of the Alfven wave's known and unknown zoo.

Note that the perpendicular wave vector component of the shear Alfven wave is related to the kinetic correction terms $\frac{\epsilon_{x x}}{\epsilon_{z z}}$ (LD), $\frac{\epsilon_{x x}}{\epsilon_{y z}}$ (TTMP) and to the ion Larmor radius correction that come from the component $\epsilon_{x x}$. This correction is finite if we use a full kinetic plasma model. The information on the Landau wave-particle interaction (growth or dissipation) is described by the anti-Hermitian part of $\epsilon_{z z}$ and the finite ion Larmor correction is contained in the Hermitian part of $\epsilon_{x x}$.

From this conjecture, it is clearly seen that only the electron mass correction is not enough to explain the necessary strong auroral electric potentials [thousands of volts], using the shear Inertial Alfven wave (IAW) as source mechanism. Indeed, the combination of finite electron mass and finite ion Larmor radius is the necessary ingredient to guarantee enough electric field intensity to explain the acceleration of electrons in aurora. If the ion Larmor radius is negligible the field aligned potential will be not zero of course, but too small. 
The kinetic Alfven wave (kAw) has no transit-time magnetic pumping and cross term contributions described in $\epsilon_{y y}$ and $\epsilon_{y z}$. The explanation is that this mode has finite $E_{z} \neq 0$ but very small $B_{z} \approx 0$.

From Equation (1), we describe:

Now we can play around with Equation (1) to name the Alfven wave as found in the literature:

\subsection{Alfven Waves Considering the Target Plasma Cold and as a Fluid $\left[\epsilon_{z z} \rightarrow+\infty\right.$ It Means That the Wave Magnetic Field Aligned Electric Field Is Short Circuited in Those Plasmas $E_{z} \rightarrow 0$ ]}

We get the regular well-known shear Alfven wave also called MHD Alfven wave [13].

For small wave number $k$ and $\sin (\theta)$ not near to zero we get,

$$
\frac{\omega^{2}}{k_{z}^{2} v_{A}^{2}} \approx 1-\frac{k^{2} c^{2}}{\omega_{p i}^{2}} \cot ^{2}(\theta)
$$

It can be seen how the ions dynamic introduces dispersivity into the shear Alfven wave dispersion relation. So, here, we just have basically the shear Alfven wave dispersion relation corrected for finite perpendicular wavelength and finite ion cyclotron frequency effects. If we assume in $\left(1^{\prime}\right)$ that $\left.\cot ^{2}(\theta) \approx 1\right)$, we have.

$$
\frac{\omega^{2}}{k_{z}^{2} v_{A}^{2}} \approx 1-\frac{k^{2} c^{2}}{\omega_{p i}^{2}} \quad \text { or } \quad \omega \approx k_{z} v_{A}\left[1-\frac{k_{\perp}^{2} c^{2}}{\omega_{p i}^{2}}-\frac{k_{z}^{2} c^{2}}{\omega_{p i}^{2}}\right]
$$

For $\cot ^{2}(\theta) \approx \frac{1}{2}$, we get a more compact form

$$
\omega \approx k_{z} v_{A}\left[1-\frac{k_{\perp}^{2} c^{2}}{2 \omega_{p i}^{2}}-\frac{k_{z}^{2} c^{2}}{2 \omega_{p i}^{2}}\right] \approx k_{z} v_{A}\left[1+\frac{k_{\perp}^{2} c^{2}}{2 \omega_{p i}^{2}}+\frac{k_{z}^{2} c^{2}}{2 \omega_{p i}^{2}}\right]^{-\frac{1}{2}}
$$

This mode is also called electromagnetic ion cyclotron wave (EMICW).

For parallel propagation ( $\theta \approx 0)$ propagation, (1.3) reads,

$$
\frac{\omega^{2}}{k_{z}^{2} v_{A}^{2}} \approx 1-\frac{v_{A}^{2}}{c^{2}} \mp \frac{\omega}{\omega_{c i}}+\frac{v_{A}^{4}}{c^{4}} .
$$

and for $\omega \ll \omega_{c i}$, we get from (1.3) the well known MHD shear Alfven wave dispersion relation.

$$
\omega^{2} \approx k_{z}^{2} v_{A}^{2} .
$$

This mode is non-dispersive and non-evanescent by nature, it is a natural "eigenmode" of magnetized homogeneous infinite plasmas. Of course it makes not much sense to talk about "Eigen" modes in an infinite plasma (not bounded), but we use this term here just to show that this mode is excitable in this type of plasma. The MHD shear Alfven wave has no electric field and no magnetic field aligned with the local background magnetic field and this mode is global by na- 
ture. It can be easily excited by internal or surface currents, Kelvin Helmoltz instability (sheared plasma flow) [28] and also creating a shear at the ambient magnetic field just by twisting the magnetic field lines somehow. For this mode,

$$
\theta=\operatorname{arctag}\left(\frac{k_{z}}{k_{\perp}}\right) \text {. }
$$

The wave number $k_{\perp}$ is undetermined (degenerated) for this mode. As mentioned by S. M. Mahajan [11], "this degeneracy with respect to $k_{\perp}$ has profound consequences." It leads to a continuous spectrum for shear Alfven waves in inhomogeneous plasma, even in a bounded system. To "regenerate" $k_{\perp}$ we have to introduce an additional effect (for instance finite electron mass or finite ion Larmor radius), and in doing that we can get $k_{\perp}$ explicitly at the shear Alfven wave dispersion relation. The group velocity for the MHD shear Alfven wave mode is $\frac{\partial \omega}{\partial k_{z}}=v_{A}$, which means that all shear modes travel with the same velocity, so a packet of shear Alfven waves do not get distorted during propagation, this feature creates a very interesting property for the shear Alfvenic turbulence in cold plasmas.

\subsection{Shear Alfven with Finite Electron Inertia $\left[m_{e} \neq 0\right]$ and Cold Ions $\left[T_{i}=0\right]$ Effects}

In this scenario the shear Alfven wave is called by some authors inertial Alfven wave (IAW). I consider this a very misleading name to the shear Alfven wave, since this is nothing more than a special case of Equation (1), it is indeed a shear Alfven wave after all. The dispersion relation for the IAW is:

$$
\omega_{k}^{2} \approx k_{z}^{2} v_{A}^{2}\left[1+\frac{k_{\perp}^{2} c^{2}}{\omega_{p e}^{2}}\right]^{-1}
$$

As said, the Inertial Alfven Wave (1.4) is just the shear Alfven wave derived from MHD equations, but to get the IAW a partial kinetic or two fluids corrections have to be considered, to bring out its propagation, polarization, and dissipation properties. What is the gain considering this correction? That is what one gets; a small magnetic field aligned wave electric field and so an explicitly determined $k_{\perp}$ as we will see below.

Of course this wave could be also called kinetic Alfven wave, finite electron mass Alfven wave or inertial Alfven wave; however it is nothing more than the shear Alfven mode in the kinetic theory context. This mode has the non-vanishing small first order electric field component, aligned to the background magnetic field, since now $\in_{z z}$ is finite implying that $E_{z} \neq 0$.

This mode is evanescent for certain ranges of plasma densities $\left[k_{\perp}^{2}<0\right]$, when $\frac{k_{z}^{2} v_{A}^{2}}{\omega^{2}}<1$, and therefore it can be excited only by a tunneled surface wave electric field, internal current sources, turbulence or by resonant mode conversion 
where $k_{\perp}^{2} \geq 0$. The group velocity for this mode is:

$$
\frac{\partial \omega_{k}}{\partial k_{z}}=v_{A} \sqrt{\left[1+\frac{k_{\perp}^{2} c^{2}}{\omega_{p e}^{2}}\right]^{-1}} .
$$

This shows that the source of the shear Alfven wave dispersivity is to consider the electron inertia finite, which is neglected at the MHD one fluid equations-ions and electrons are not distinct.

\subsection{Shear Alfven Wave with Quasi-Cold (Cold Ion and Warm} Electrons) $\left[T_{i}=0\right]\left[T_{e} \neq 0\right]$ and Finite Electron Inertia

$$
\left[m_{e} \neq 0\right]\left[m_{i} \neq 0\right]\left[\rho_{s}^{2}=\frac{v_{s}^{2}}{\omega_{c i}^{2}} \neq 0\right]
$$

In this case Equation (1) leads to:

$$
\omega_{k}^{2} \approx k_{z}^{2} v_{A}^{2}\left[1+\frac{3}{4} k_{\perp}^{2} \rho_{s}^{2}\right]\left[1+\frac{k_{\perp}^{2} c^{2}}{\omega_{p e}^{2}}\right]^{-1}
$$

where now the "Larmor radius" is emulated by $\rho_{s}^{2}=\frac{v_{s}^{2}}{\omega_{c i}^{2}}$, since $v_{t h i}^{2}=0$.

This mode could be easily called Inertial Alfven wave with finite electron temperature correction and finite ion cyclotron frequency correction. If $T_{e} \rightarrow 0$, we have also $\rho_{s} \rightarrow 0$ and so we recover the so called Inertial Alfven wave independent of the value of $\omega_{c i}$.

The factor $k_{\perp}^{2} \rho_{s}^{2} \quad\left[k_{\perp} \rho_{s}=k_{\perp} \sqrt{\frac{T_{e}}{m_{i}}} / \omega_{c i}\right]$ is due to the electron temperature correction and it plays a similar role as in the finite ion Larmor radius correction. The factor $\frac{k_{\perp}^{2} c^{2}}{\omega_{p e}^{2}}$ is due to the electron inertia. The relative size of these two corrections can be evaluated by observing that $\frac{\rho_{s}^{2}}{\frac{c^{2}}{\omega_{p e}^{2}}}=\frac{m_{e}}{m_{i}} \beta$.

For $\beta\left[=\frac{v_{t h i}^{2}}{v_{A}^{2}}=\frac{m_{e}}{m_{1}} \frac{v_{t h e}^{2}}{v_{A}^{2}}\left(T_{e}=T_{i}\right)\right]$ larger than $\frac{m_{e}}{m_{i}}$ the finite Larmor radius correction dominates and vice versa. If $\beta \ll \frac{m_{e}}{m_{i}}$, we have: $\omega^{2} \approx k_{/ /}^{2} v_{A}^{2}\left[1+\frac{c^{2} k_{\perp}^{2}}{\omega_{p e}^{2}}\right]^{-1}$ and the propagation is allowed in the large $v_{A}$ region, whereas when $\beta \gg \frac{m_{e}}{m_{i}}$, the reverse is true and the dispersion relation (1.5) becomes: $\omega^{2} \approx k_{z}^{2} v_{A}^{2}\left[1+k_{\perp}^{2} \rho_{s}^{2}\right]$.

These cases can be already obtained in the MHD approximation using the modified Ohm's Law, however in the MHD model it is not possible to account for properly the appearance of the mode dissipation (Landau damping). 


\subsection{Shear Alfven Wave with Relatively Cold Plasma [lukewarm Ions $T_{i} \neq 0$ and Warm Electrons $\left.T_{e} \neq 0\right]\left[v_{\text {the }}<v_{A}\right]$}

In this case the electron inertia becomes important and the dispersion relation reads - which is the Equation (1.5):

$$
\omega_{k}^{2} \approx k_{z}^{2} v_{A}^{2}\left[1+\frac{3}{4} k_{\perp}^{2} \rho_{i}^{2}\right]\left[1+\frac{k_{\perp}^{2} c^{2}}{\omega_{p e}^{2}}\right]^{-1} .
$$

The hot low $\beta\left[v_{s}^{2} \ll v_{A}^{2}\right]$ small Larmor radius plasma shear Alfven wave Assuming that $k_{\perp}^{2} \rho_{i}^{2} \ll 1$, we find for (1.5):

$$
\omega_{k}^{2}=k_{z}^{2} v_{A}^{2}\left[1+\left[\frac{3}{4}+\frac{T_{e}}{T_{i}}\right] k_{\perp}^{2} \rho_{i}^{2}\right]
$$

This mode is already labeled as kinetic shear Alfven wave.

\subsection{Shear Alfven Wave in Hot Low $\beta_{\text {plasmas }}\left[v_{s}^{2} \ll v_{A}^{2}\right]$ and Finite Ion Larmor Radius Plasma}

In this case the shear Alfven wave has the dispersion relation described also by Equation (1), that is,

$$
\Omega_{/ /}^{2}\left(k_{/ /}, k_{\perp}\right)=k_{/ /}^{2} v_{A}^{2} I\left(k_{\perp}\right)
$$

with,

$$
I\left(k_{\perp}\right)=\frac{k_{\perp}^{2} \rho_{i}^{2}}{1-I_{0}\left(k_{\perp}^{2} \rho_{i}^{2}\right) \exp \left(-k_{\perp}^{2} \rho_{i}^{2}\right)}+\frac{T_{e}}{T_{i}} k_{\perp}^{2} \rho_{i}^{2}
$$

In (1.8), $I_{0}$ is the modified Bessel's function of zeroth order.

In (1.7) the Larmor radius is kept finite, no expansion is made. This means that this is the full kinetic shear Alfven wave. This dispersion relation reproduces, taking the appropriate limits, both; the Inertial Alfven Wave and the Kinetic Alfven Wave and, of course, all the intermediates nameless modes.

And so, the kinetic Alfven wave is just the same shear Alfven wave derived from MHD equations, but considering the kinetic approach. It is still the shear Alfven wave, but now both finite ion Larmor radius and finite electrons mass (inertia) corrections are considered to derive its propagation, polarization and dissipation properties.

The name kinetic Alfven wave "appears" when the dispersion relation of the shear Alfven wave contains the finite electron mass and the finite ion Larmor radius corrections, this name was first used by Akira Hasegawa [16] in the context of inhomogeneous plasmas. However, this dispersion relation can easily be obtained from the Alfven's dielectric tensor/dispersion relation, for homogeneous plasmas, as we have shown. In high beta plasmas the kinetic Alfven wave is coupled to the ion acoustic wave.

Now, we consider the ion Larmor radius finite, no expansion is made. This means that this is the full shear kinetic Alfven wave dispersion relation. This 
dispersion relation contemplates, taking the appropriate limits, both; the so called Inertial Alfven Wave (IAW) and the kAw.

When $I=0$ we recover the dispersion relation for the MHD shear Alfven wave, it means when

$$
T_{e}=\frac{T_{i}}{I_{0}\left(k_{\perp}^{2} \rho_{i}^{2}\right) \exp \left(-k_{\perp}^{2} \rho_{i}^{2}\right)-1} .
$$

Since,

$$
\frac{\omega_{k}^{2}}{k_{z}^{2} v_{A}^{2}}=I^{2}\left(k_{\perp}\right)
$$

Depending on the signal of $\frac{\omega_{k}^{2}}{k_{z}^{2} v_{A}^{2}}$ the wave can be evanescent. This is directly related to the background magnetic field and plasma density. When this happens $k_{\perp} \rightarrow j k_{\perp}\left(k_{\perp}^{2}<0\right)$ where $j=\sqrt{-1}$. This must always be checked before we can say that we have observed a shear Alfven wave. This is more complex in non-homogeneous and layered plasma where surface wave is normally present and has propagation property not so different as the shear Alfven at the field aligned background magnetic field direction.

The group velocity is given by,

$$
\frac{\partial \omega_{k}}{\partial k_{z}}=v_{A} I\left(k_{\perp}\right) .
$$

Equation (1.9) shows how dispersive is the kAw and again the dispersivity is due to the electron inertia and due to the additional term related to the finite corrections of the ion Larmor radius.

Since aurora is highly populated with current systems, it is in order to ask how background currents affect the shear Alfven wave dispersion relation. The Current carrying shear Alfven wave (called in nuclear fusion by global Alfven wave, GAE, TAE et cetera) is nothing more than the well-known shear Alfven mode where a local dc current system is present (toroidal, dipolar et cetera)-for aurora dynamics the current might be the dc current system aligned with the ambient magnetic field. The current creates a poloidal dc magnetic field (emulated by the $y$-direction in our coordinate system), which modifies directly the known shear Alfven wave dispersion relation derived from the non-current carrying MHD plasma.

What we gain considering current carrying plasmas? First of all, the current system creates a dispersivity. The current carrying mode can be excited directly (without the need of surface wave mode conversation) by any shear or compression in the ambient magnetic field. It propagates with certain level of dissipation, due to a small but finite magnetic field aligned electron field and magnetic field, very similar situation to the compressional case. This mode gets some compressive property due to $k_{\perp}$, which is undetermined (degenerated) in the case of a shear Alfven mode derived from pure MHD equations. In this way, this mode 
can be dissipated by either Landau damping or TTMP or by both depending on the situation

\subsection{The Alfven Surface Wave}

There are two types of Alfven surface wave (Asw), the shear-type (non compressive) [22] and the compressional [21]. As I have already mentioned, the Asw are natural eigenmodes of sharp density boundaries magnetized plasmas, and evanescent eigenmodes of smooth density boundaries magnetized plasmas [21] [22] [27]. For the smooth-boundary condition, surface waves can very efficiently couple energy to the kAw via the resonant mode conversion. The Asw can be easily confused with the kinetic Alfven wave if the polarization properties of the observed turbulence are not looked at carefully, and this is due to its frequency being close to the bulk shear Alfven wave frequency, i.e.;

$$
\begin{aligned}
& \omega_{s}^{2} \approx 2 k_{z}^{2} v_{A}^{2} \\
& \frac{\partial \omega_{s}}{\partial k_{z}}=\sqrt{2} v_{A}
\end{aligned}
$$

The group velocity (1.9') of the surface wave (2) is slightly larger than that of the shear ideal Alfven wave, however, again, the all modes at the wave packet travel with the same constant group velocity $\sqrt{2} v_{A}$ at this approximation.

In order to introduce the final clarification to this issue, now, if one looks at Stix's book [14] one can see that the left-hand cut-off, the right-hand cut-off, the Alfven resonance, the surface wave, the fast magnetosonic wave and the kinetic Alfven wave are closely related. Stix just plots the perpendicular index of refraction $n_{\perp}^{2}=\frac{k_{\perp} c^{2}}{\omega^{2}}$ versus plasma density.

He gets the following interesting results and final results to this Alfven wave ensemble name problem:

1) Starting from low density side, the square of the refraction index plotted in this case for the compressional Alfven waves according to cold-plasma (two-fluid) theory shows that for increasing density $n_{\perp}^{2}$ evolves from an evanescent $\left(n_{\perp}^{2}<1\right)$ mode to a propagating one at the left-hand cut-off point $n_{/ /}^{2}=L$. However, it diverges at the Alfven resonance $n_{/ /}^{2}=S$. Starting from higher density side $n_{\perp}^{2}$ evolves from a propagating mode to an evanescent mode exactly at the Alfven resonance $n_{/ /}^{2}=S$, so both compressional modes (low frequency and high frequency) are evanescent at $n_{/ /}^{2}=S$.

2) Incorporating now, the finite-Larmor-radius corrections (but zero electron temperature), from the hot-plasma theory, to the dispersion relation. In doing so, Stix has gotten for this case (called "cool") a short-wavelength propagating mode on the low density side of the resonance and he called it "surface wave". This new situation does not show any singular behaviour at the Alfven resonance region. It means that the surface modes comes from low density side as evanescent up to close to the left-hand cut-off region and then propagate back to the lower density side. The fast mode propagates from the higher density side to the lower 
density side and just after the right-hand cut-off it becomes an evanescent mode.

3) Incorporating into the dispersion relation both; the finite-Larmor-radius and finite electron temperature corrections, using the hot-plasma theory, Stix has gotten for this case (called "warm") a surprising short-wavelength propagating mode on the higher density side of the resonance. This mode propagation on the higher density side is labelled by other people kinetic Alfven wave. Since it is somehow related to the previous lower density propagating modes, it is evanescent for plasma densities where we have the match $n_{/ /}^{2}=S$.

Going further with this analysis, Stix mention that the finite electron temperature has a remarkable effect similar to ion temperature in changing the wave "cool" wave behavior to "warm" wave behavior. And going further he says that whereas raising the ion temperature increases the finite-Larmor-radius effects, raising the electron temperature so that changes sign of the $z z$ dielectric tensor component $\epsilon_{z z}$.

The Alfven shear mode has only $k_{z}$ when derived from MHD equations. However, if kinetic corrections are included a perpendicular component $k_{\perp}$ appears and the dispersion relation becomes

$$
\omega_{k}^{2}=v_{A}^{2}\left[k_{z}^{2}+k_{\perp}^{2}\right]
$$

Note that the electron dynamic along the magnetic field lines gives rise to a finite field aligned electric field $E_{z}$, in both Alfven modes namely the shear mode and the compressional mode described by (3). For the compressional mode this electric field is related to the wave field aligned magnetic field $B_{z}$ through $E_{z}=j \frac{k_{z} c}{c \omega_{c e}} B_{z}$.

It is important to note that the Alfven surface wave and kinetic Alfven wave are coupled in non-homogeneous kinetic plasmas, in this kinetic plasma model the differential equation that describes the electric field in the direction of the non-homogeneity is of fourth order and the coupling is due to the finite ion Larmor radius kinetic correction. Surface waves in inhomogeneous plasmas are evanescent in the direction of the plasma non homogeneity. For sharp nonhomogeneity jump the surface wave is a propagating mode in the direction of the ambient magnetic field but it is evanescent in the direction of the plasma nonhomogeneity. So, the electric field in the direction of the plasma gradient is localized at a distance inside the plasma of $d \approx \frac{2 \pi}{k_{x}}$.

Due to the nature of the surface wave and the kinetic Alfven the former can couple energy to the latter. Since, as we have seen, the surface wave is an excitable eigenmode at the lower density side of the plasma and the kinetic Alfven is evanescent at the lower density side of the plasma and cannot be excited directly at this region, the connection between surface waves and kinetic Alfven can guarantee the energy coupling from surface to kinetic Alfven wave, what is called in kinetic theory resonant mode conversion. 
Here,

$$
n_{\perp}^{2}=\frac{\left(R-n_{/ /}^{2}\right)\left(L-n_{/ /}^{2}\right)}{S-n_{/ /}^{2}}
$$

where,

$$
\begin{gathered}
R=1+\gamma \frac{\omega_{c i}}{\omega_{c i}+\omega}, \\
L=1+\gamma \frac{\omega_{c i}}{\omega_{c i}-\omega}, \\
S=1+\gamma \frac{\omega_{c i}^{2}}{\omega_{c i}^{2}-\omega^{2}}, \\
\gamma=\frac{\omega_{p i}^{2}}{\omega_{c i}^{2}}=\frac{c^{2}}{v_{A}^{2}} .
\end{gathered}
$$

It is interesting what Hollweg [30] says about the kinetic Alfven wave: "the wave becomes strongly compressive when $k_{\perp}^{-1}$ is of the order of the ion inertial length. Thus, in low- $\beta$ plasma, the kinetic Alfven wave can be compressive at values of $k_{\perp}$ for which the dispersion relation departs only slightly from that of the usual MHD Alfven wave. The compression is accompanied by a magnetic field fluctuation $\delta B_{/ /}$such that the total pressure perturbation $\delta p_{\text {tot }} \approx 0$. Thus the wave undergoes transit-time damping as well as Landau damping; the two effects are comparable if the ion thermal speed is of the order of the Alfven speed. We find that the transverse electric field is elliptically polarized but rotating in the electron sense; this surprising behaviour of the polarization of the Alfven branch was discovered numerically by Gary [31]".

It is also interesting that Holweg [30] explicitly shows how the kinetic Alfven wave takes on some properties of the large- $k_{\perp}$ limit of the slow mode, as can also be seen from (4).

\section{Conclusion}

The Alfven wave is, with no caveat, the most interesting eigenmode for magnetized plasmas. They can be externally excited to heat and drive non-inductive current in fusion plasmas and also in space and astrophysical plasmas. They can accelerate ions and electrons, induce magnetic reconnections, and other remarkable effects in space. These modes can also couple to each other and with other different plasma wave modes, and therefore they can excite other frequency range modes such as ion acoustic waves and whistler waves. Alfven waves can exhibit so interesting behaviours that even now after so many years of its discovery it is still fascinating and it is challenging plasma physicist, from the different plasma communities; as from astrophysics, stellar, space, fusion, and technological areas, to still study and enjoy them [11] [14] [15] [17]-[24]. 


\section{Acknowledgements}

The authors (A. S. de Assis and C. E. da Silva) would like to thank Yahweh for granting us the beauties of knowledge and a learning spirit.

\section{Conflicts of Interest}

The authors declare no conflicts of interest regarding the publication of this paper.

\section{References}

[1] Alfvén, H. (1942) Existence of Electromagnetic-Hydrodynamic Waves. Nature, 150, 405-406.

Alfvén, H. (1942) On the Existence of Electromagnetic-Hydrodynamic Waves. Arkiv för Matematik, Astronomi och Fysik B, 29, 1942.

Alfvén, H. (1950) Cosmic Electrodynamics. Oxford University Press, New York.

Alfvén, H. (1988) Plasma and the Universe. Fälthammar, C.-G., de Bibhas, R., Arrhenius, G., Herlofson, N., Mendis, D.A. and Kopal, Z., Eds., Springer, The Netherlands.

[2] Fermi, E. (1949) On the Origin of the Cosmic Radiation. Physical Review, 75, 1169-1174. https://doi.org/10.1103/PhysRev.75.1169

[3] Lehnert, B. (1954) Magneto-Hydrodynamic Waves in Liquid Sodium. Physical Review, 94, 815-824. https://doi.org/10.1103/PhysRev.94.815

[4] Lundquist, S. (1949) Experimental Investigations of Magneto-Hydrodynamic Waves. Physical Review, 76, 1805-1809. https://doi.org/10.1103/PhysRev.76.1805

[5] Parker, E.N. (1955) Hydromagnetic Waves and the Acceleration of Cosmic Rays. Physical Review, 99, 241-253. https://doi.org/10.1103/PhysRev.99.241

[6] Ip, W.-H. and Mendis, D.A. (1975) The Cometary Magnetic Field and Its Associated Electric Currents. Icarus, 26, 457-461.

https://doi.org/10.1016/0019-1035(75)90115-3

[7] Coleman, P.J., Sonett, C.P., Judge, D.L. and Smith, E.J. (1960) Some Preliminary Results of the Pioneer V Magnetometer Experiment. Journal of Geophysical Research, 65, 1856-1857. https://doi.org/10.1029/JZ065i006p01856

[8] Berthold, W.K., Harris, A.K. and Hope, H.J. (1960) World-Wide Effects of Hydromagnetic Waves Due to Argus. Journal of Geophysical Research, 65, 2233-2239. https://doi.org/10.1029/JZ065i008p02233

[9] Arker, E.N. (1973) Extragalactic Cosmic Rays and the Galactic Magnetic Field. Astrophysics and Space Science, 24, 279. https://doi.org/10.1007/BF00648691

[10] Hollweg, J.V. (1974) Hydromagnetic Waves in Interplanetary Space. Publications of the Astronomical Society of the Pacific, 86, 561-594. https://doi.org/10.1086/129646

[11] Mahajan, S.M. (1995) Spectrum of Alfven Wave, a Brief Review. Physica Scripta, 51, 1-11. https://doi.org/10.2172/108117

[12] Shafranov, V.D. (1965) Reviews of Plasma Physics. 3rd Edition, Consultants Bureau, New York.

[13] Akhiezer, A.I., Akhiezer, I.A., Polovin, N., Sitenko, S. and Stepanov, R.N. (1975) Plasma Electrodynamics, Volumes 1 and 2. Pergamon Press, London. https://doi.org/10.1016/B978-0-08-017783-0.50005-1

[14] Stix, T.H. (1992) Waves in Plasmas. American Institute of Physics, College Park.

[15] Hasegawa, A. and Uberoi, C. (1982) The Alfven Wave, DOE Critical Review Series, 
DOE/TIC-11197. Technical Information Center, U.S. Department of Energy, Springfield. https://doi.org/10.2172/5259641

[16] Hasegawa, A. and Chen, L. (1974) Plasma Heating by Alfvén-Wave Phase Mixing. Physical Review Letters, 32, 454. https://doi.org/10.1103/PhysRevLett.32.454

[17] Vaclavik, J. and Appert, K. (1993) Nuclear Fusion 21, 10.

[18] Appert, K., Vaclavik, J. and Villard, L. (1984) Spectrum of Low-Frequency Nonaxisymetric Oscillations in a Cold, Current-Carrying Plasma Column. Physics of Fluids, 27, 432-437. https://doi.org/10.1063/1.864638

[19] Yoon, P.H., Wu, C.S. and De Assis, A.S. (1993) Effect of Finite Ion Gyroradius on the Fire-Hose Instability in a High Beta Plasma. Physics of Fluids B: Plasma Physics, 5, 1971-1979. https://doi.org/10.1063/1.860785

[20] De Assis, A.S. and Busnardo-Neto, J. (1987) Cherenkov Damping of Surface Waves. Astrophysical Journal, 323, 399-405. https://doi.org/10.1086/165837

[21] De Assis, A.S. and Tsui, K.H. (1991) Cherenkov Damping of Surface Waves. Astrophysical Journal, 324, 327-405.

[22] Hasegawa, A., Tsui, K.H. and de Assis, A.S. (1983) A Theory of Long Period Magnetic Pulsations, 3. Local Field Line Oscillations. Geophysical Research Letters, 10, 765-767. https://doi.org/10.1029/GL010i008p00765

[23] Halberstadt, G. and Goedbloed, J.P. (1995) Alfven Heating of Line-Tied Loops. Astronomy and Astrophysical, 301, 577-592.

[24] Elfimov, A.G., de Azevedo, C.A. and de Assis, A.S. (1996) Non Inductive Current Driven by Alfven Waves in Solar Coronal Loops. Solar Physics, 167, 203-216. https://doi.org/10.1007/BF00146337

Elfimov, A.G., de Azevedo, C.A. and de Assis, A.S. (1994) Coronal Loop Heating by Alfven Waves. Solar Physics, 153, 205-215. https://doi.org/10.1007/BF00712502

[25] Kitamura, T. (1998) How Does the Ionosphere Work on ULF? In: de Assis, A.S. and de Azevedo, C.A., Eds., International Workshop on Magnetospheric Plasmas Proceedings, State University of Rio de janeiro (UERJ) and XEROX, Rio de janeiro.

[26] Stasiewicz, K., et al. (2000) Small Scale Alfvénic Structure in the Aurora. Space Science Reviews, 92, 423-533. https://doi.org/10.1023/A:1005207202143

[27] Hasegawa, A. (1976) Particle Acceleration by MHD Surface Waves and Formation of Aurora. Journal of Geophysical Research, 81, 5083-5090. https://doi.org/10.1029/JA081i028p05083

[28] Ogilvie, K.W. and Fitzenreiter, R.J. (1989) The Kelvin-Helmholtz Instability at the Magnetopouse and Inner Boundary Layer Surface. Journal of Geophysical Research, 94, 15113-15123. https://doi.org/10.1029/JA081i028p05083

[29] Paschmann, G., Haaland, S. and Teeumann, R. (2003) Auroral Plasma Physics. Kluwer Academic Publishers, Space Sciences Series of ISSI, London. https://doi.org/10.1007/978-94-007-1086-3_1

[30] Holweg, J.V. (1999) Kinetic Alfvén Waves Revisited. Journal of Geophysical Research, 104, 14811-14819.

[31] Gary, S.P. (1986) Low-Frequency Waves in High Beta Collisionless Plasma: Polarization, Compressibility and Helicity. Journal of Plasma Physics, 35, 431-447. https://doi.org/10.1017/S0022377800011442 